\title{
Ray tracing methods in numerical analysis of double-clad microstructured optical fibre coupler
}

\author{
L. Pajewski ${ }^{1}$ (D) H. Stawska ${ }^{1} \cdot$ E. Beres-Pawlik ${ }^{1}$
}

Received: 6 October 2016/Accepted: 4 February 2017/Published online: 11 February 2017

(C) The Author(s) 2017. This article is published with open access at Springerlink.com

\begin{abstract}
The article presents the analysis of a double-clad microstructured optical fibre (DC MOF) couplers based on the ray tracing technique. The calculations were done for couplers made of well-defined fibre structures. Such couplers can be employed in laser systems using a DC MOF as a gain medium and a side pumping method which will allow to build fully-fiberized laser systems based on active microstructured fibres.
\end{abstract}

Keywords Microstructured couplers $\cdot$ Ray tracing $\cdot$ Optical fibre $\cdot$ Side-polishing

\section{Introduction}

Thanks to their designable structural and waveguide parameters, microstructured optical fibres have found numerous applications in contemporary photonics. Due to the possibility of strong interference in their structure, it is possible to develop optical fibres with parameters strongly varied from conventional fibres. As a result they are frequently used in high-power laser systems in which active optical fibres are used.

For the purpose of obtaining high output power in optical fibre lasers, it is necessary to maintain the power saturation of the core along its whole length. The pumping method and the construction of the active fibre are of decisive significance here. In the case of singleclad fibres, end-pumping results in a decrease of the pumping power along with an increase

This article is part of the Topical Collection on Optical Wave and Waveguide Theory and Numerical Modelling 2016.

Guest edited by Krzysztof Anders, Xuesong Meng, Gregory Morozov, Sendy Phang, and Mariusz Zdanowicz.

L. Pajewski

lukasz.pajewski@pwr.edu.pl

1 Wroclaw University of Science and Technology, Wrocław, Poland 
in the distance from the pumping point. Coupling the pump into the single-clad fibre becomes even more difficult, when one considers the small values of the numerical aperture (NA). The solution to this problem is the use of double-clad (DC) fibres. Thanks to the application of second cladding with a high NA, an easier introduction of the pumping power to a core and exciting it on a much longer section is possible.

As it has already been mentioned, one of the ways of pumping active fibres is endpumping. In the case of high-power lasers, usually many pumps or array modules are used. Their introduction to the fibre requires the use of bulk optics, tapers or fibre combiners. Another method of DC fibre pumping is side-pumping. According to (Zervas and Codemard 2014) the most common methods employed in side-pumping are: pumping using a $\mathrm{V}$-groove milled in the cladding, angle-polished or tapered pump fibre attached to or fused into the cladding of the signal fibre, and pumping based on a multi-fibre assembly in optical contact surrounded and held together by a common low-index polymer cladding.

In standard fibres, the side-pumping method is widely used, but it is not developed for MOF laser cavities yet. The utilization of the side-pumping method allows to design the all-fibre laser cavity. In this paper side-pumping can be achieved by using side-polish couplers made of active MOF and the multimode pumping fibre. This method was successfully used in standard DC fibre laser cavities (Beres-Pawlik and Grobelny 2010; BeresPawlik 2014) also the first non-DC MOF coupler was made using the side-polishing technique (Kim et al. 2004). For the purpose of the implementation of this method, it is necessary to determine the construction parameters of optical couplers made of microstructured and conventional optical fibres, such as: polishing depth, the bending radius of particular fibres, shifting of optical fibres against one another and the thickness of the matching gel layer (Fig. 1).

Optimising the values of those parameters is necessary to achieve good pumping parameters, which would allow to reduce the insertion losses and, therefore, increase the amount of pump power coupled into the fibres inner cladding. Due to the high cost of MOF, optimisation of those parameters through trial and error would be highly uneconomic. Therefore, it is necessary to find a method to simulate the effect of mentioned

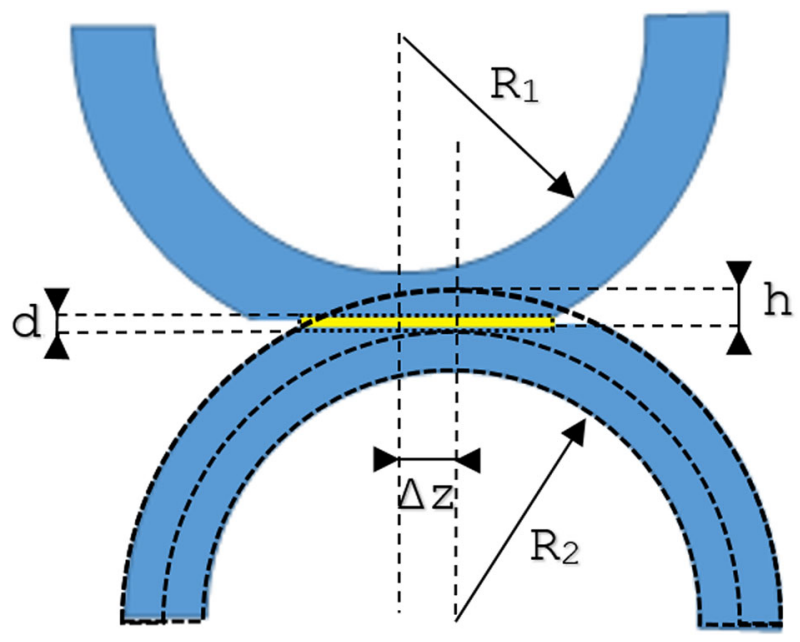

Fig. 1 Coupler design: R1,R2—-bending radii, $\mathrm{h}$-polishing depth, $\Delta \mathrm{z}$ - shifting of optical fibres against one another, $\mathrm{d}$-thickness of the matching gel layer 
parameters on the couplers pumping efficiency. The proposed solution is to use ray tracing method. This method is well known and used, including the bulk optics systems (Horiuchi et al. 2010) and for analysis the light extraction efficiency of LEDs (Borbely and Johnson 2004; Lalau Keraly et al. 2013; Zang et al. 2016). The ray tracing method based on the geometrical optics allows to determine of the route travelled by the ray from the moment of launch, by using the Snell's law. To determine the parameters of complex optical systems, such as couplers, the ray tracing method in combination with the Monte Carlo method can be used. The authors show that by assuming a distribution of rays at the end-face of the fibre and analysing the amount of rays that have been guided to MOF, which depending on the chosen construction parameters of the coupler, it is possible to determine the optimal construction parameters for which the maximum quantity of radiation passes from the pumping arm to the active optical fibre. In the literature, to the best knowledge of the authors of this article, the optimisation of couplers parameters (made by pumping fibre and active DC MOF) by using the ray tracing method has never been given: only particular cases have been given. In Beres-Pawlik and Grobelny (2010) Beres-Pawlik and Grobelny used this method to the analysis of DC couplers but they were interested only in a nonmicrostructure cases.

\section{Principles}

This article presents the ray-tracing method in which every next step is determined on the basis of the previous one and the radius parameters (Heimrath 1988). There is also another method in which the basis for the next step are the radius parameters and two intermediate steps (Sharma et al. 1982).

Both methods are based on the same principle and describe the ray-tracing in media with a variable refractive index:

$$
\frac{\mathrm{d}}{d s}\left[n(r) \frac{\mathrm{d} r}{d s}\right]=\nabla n(r)
$$

where: ds-elementary section of the ray, $n$-refractive index, $r$-position vector of a point on the ray. The first approach (Heimrath 1988) assumes that, the next position of the ray can by calculated by:

$$
\begin{gathered}
x_{k+1}=x_{k}+\Delta s \cos \alpha_{k} \\
y_{k+1}=y_{k}+\Delta s \cos \beta_{k} \\
z_{k+1}=z_{k}+\Delta s \cos \gamma_{k} \\
(\cos \alpha)_{k+1}=\left(\frac{1}{n}\left\{n_{x}-\left(\left[n_{x}, n_{y}, n_{z}\right] \cdot[\cos \alpha, \cos \beta, \cos \gamma]\right) \cos \alpha\right\}\right)_{k} \Delta s+(\cos \alpha)_{k} \\
(\cos \beta)_{k+1}=\left(\frac{1}{n}\left\{n_{y}-\left(\left[n_{x}, n_{y}, n_{z}\right] \cdot[\cos \alpha, \cos \beta, \cos \gamma]\right) \cos \beta\right\}\right)_{k} \Delta s+(\cos \beta)_{k}
\end{gathered}
$$




$$
(\cos \gamma)_{k+1}=\sqrt{1-(\cos \alpha)_{k+1}^{2}-(\cos \beta)_{k+1}^{2}}
$$

where:

$$
\left[n_{x}, \quad n_{y}, n_{z}\right]=\left[\begin{array}{lll}
\frac{\partial n}{\partial x}, & \frac{\partial n}{\partial y}, & \frac{\partial n}{\partial z}
\end{array}\right]=\operatorname{grad}(n)
$$

The second approach (Sharma et al. 1982) defined new variables:

$$
\begin{gathered}
d t=\frac{d s}{n}, \quad T=\frac{d r}{d t} \\
R=\left(\begin{array}{l}
x \\
y \\
z
\end{array}\right), \quad T=\left(\begin{array}{l}
T_{x} \\
T_{y} \\
T_{z}
\end{array}\right)=n\left(\begin{array}{c}
d x / d s \\
d y / d s \\
d z / d s
\end{array}\right) \\
D=n\left(\begin{array}{c}
\partial n / \partial x \\
\partial n / \partial y \\
\partial n / \partial z
\end{array}\right)=\frac{1}{2}\left(\begin{array}{l}
\partial n^{2} / \partial x \\
\partial n^{2} / \partial y \\
\partial n^{2} / \partial z
\end{array}\right)
\end{gathered}
$$

so Eq. 1 can be written as:

$$
\frac{\mathrm{d}^{2} R}{d t^{2}}=D(R)
$$

These formulas can be solved using the Runge-Kutta algorithm:

$$
\begin{gathered}
R_{n+1}=R_{n}+\Delta t\left[T_{n}+\frac{1}{6}(A+2 B)\right] \\
T_{n+1}=T_{n}+\frac{1}{6}(A+4 B+C)
\end{gathered}
$$

where:

$$
\begin{gathered}
A=\Delta t D\left(R_{n}\right) \\
B=\Delta t D\left(R_{n}+\frac{\Delta t}{2} T_{n}+\frac{1}{8} \Delta t A\right) \\
C=\Delta t D\left(R_{n}+\Delta t T_{n}+\frac{1}{2} \Delta t B\right)
\end{gathered}
$$

For the purpose of our simulations presented later, we have used technique shown in (Heimrath 1988). However, it should be noted that both methods offer consistent results, therefore any of them can be used conveniently. To define the convergence of the method as a function of the elementary step Eq. 18 was used and the results are shown in Fig. 2. 

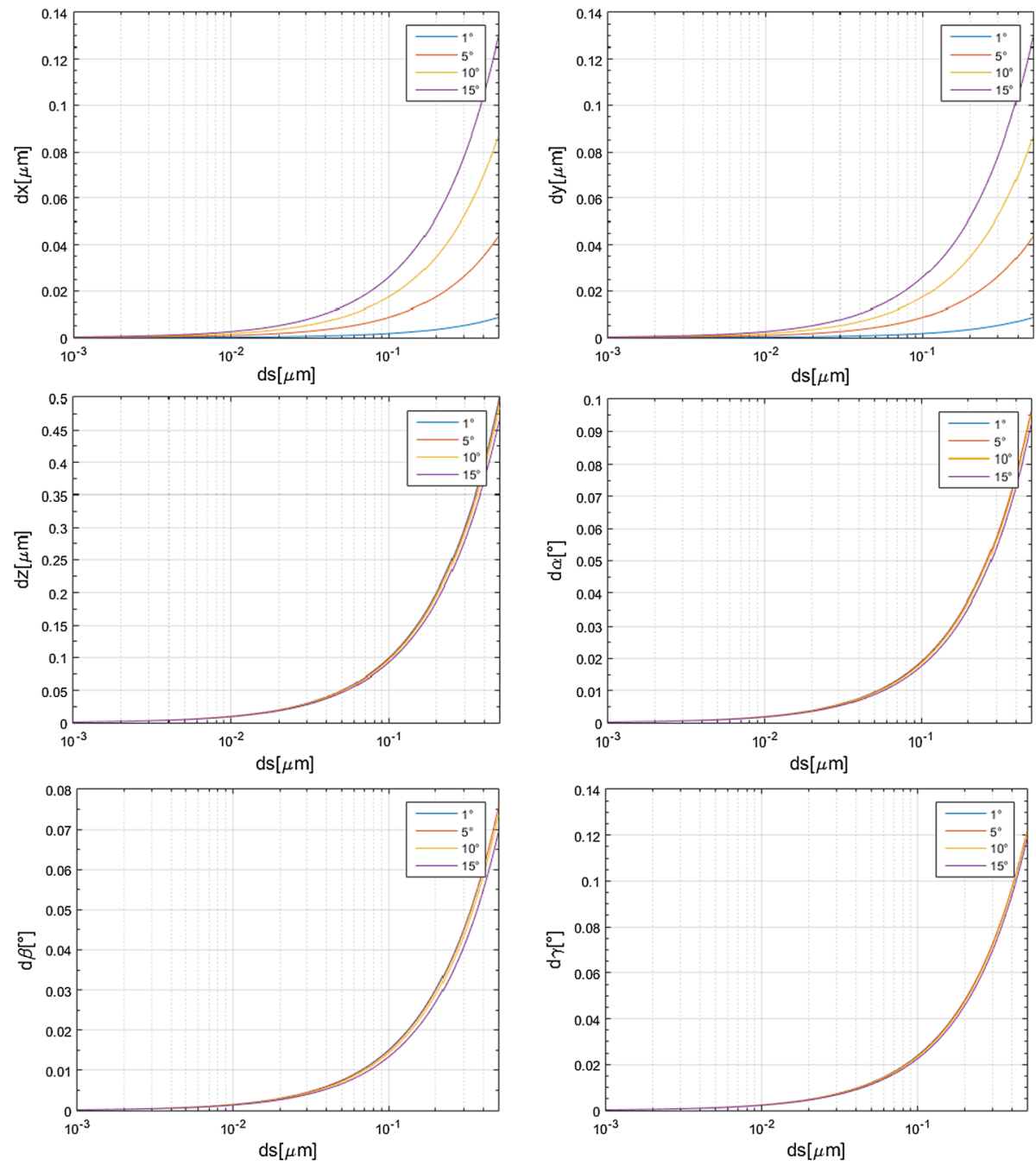

Fig. 2 Convergence of the ray tracing method as a function of an elementary step (ds). Particular graphs show the change of position $(d x, d y, d z)$ or angle $(d \alpha, d \beta, d \gamma)$ after a ray covers the length of one elementary step depending on the initial introduction angle

$$
d x(d s)=a b s\left(x_{i}-x_{i-1}\right)
$$

The values of $d y, d z, d \alpha, d \beta, d \gamma$ were determined analogically.

It can be observed that with the decrease of $d s$, the values of $d x, d y, d z, d \alpha, d \beta, d \gamma$ approximate zero. Hence, the smaller ds is accepted the better. For the purpose of achieving accurate results with simultaneous limitation of the calculation power, necessary to conduct a simulation, it was assumed that the elementary step used during the simulation will be $\mathrm{ds}=0.01 \mu \mathrm{m}$. For this value $\mathrm{dx}, \mathrm{dy}, \mathrm{dz}, \mathrm{d} \alpha, \mathrm{d} \beta, \mathrm{d} \gamma<0.025$, which was considered an acceptable error. 


\section{Experiment}

For the purpose of modelling ray-tracing inside an optical coupler made of a conventional coupler and a DC PCF, the effective refractive index theory was used. Thanks to the determination of the effective refractive index for particular microstructured fibre layers, the numerical model was simplified. Instead of numerous crossings of the area with a step change in the refractive index, the existence of a few layers with a step change of the refractive index was assumed. The effective refractive index for these layers was calculated using eigensolver implemented in Lumerical Mode Solution, commercially available software.

For the purpose of comparing the real (Fig. 3) and approximated structures, the power distribution and effective refractive indexes for the fundamental mode $(1064 \mathrm{~nm})$ of the above mentioned structures were calculated. The power distributions which are presented in Fig. 4. Figure 4a shows the power distribution of the fundamental mode of optical fibre with a real structure while Fig. $4 \mathrm{~b}$ presents the power distribution for the main optical fibre with a layered structure. Both fibres guide the fundamental mode at $1064 \mathrm{~nm}$ wavelength.

The simulated effective refractive index for the real fundamental mode was $\mathrm{n}_{\text {eff }}=1.399760$ while the same value for the approximated fibre was $n_{\text {eff }}=1.399834$. Thus, the approximation error is at the level of $\Delta \mathrm{n}_{\mathrm{eff}}=0.000074$. It can be assumed that proposed method convey the real structure with acceptable approximation.

It was also assumed that the refractive index changed smoothly between subsequent layers. Thanks to the approximation of the refractive index according to the following formula:

$$
n(r)=n_{k+1}\left\{1+\frac{n_{k}-n_{k+1}}{n_{k}} \exp \left[-\left(\frac{r^{2}}{R_{k}^{2}}\right)^{20}\right]\right\}
$$

where $\mathrm{k}$ and $\mathrm{k}+1$ indexes define between which layers the refractive index is calculated, the function $\mathrm{n}(\mathrm{x}, \mathrm{y}, \mathrm{z})$ is continuously differentiable, particularly on the border between the core and the clad.

Fig. 3 Double-clad microstructured optical fibre structure used during simulations

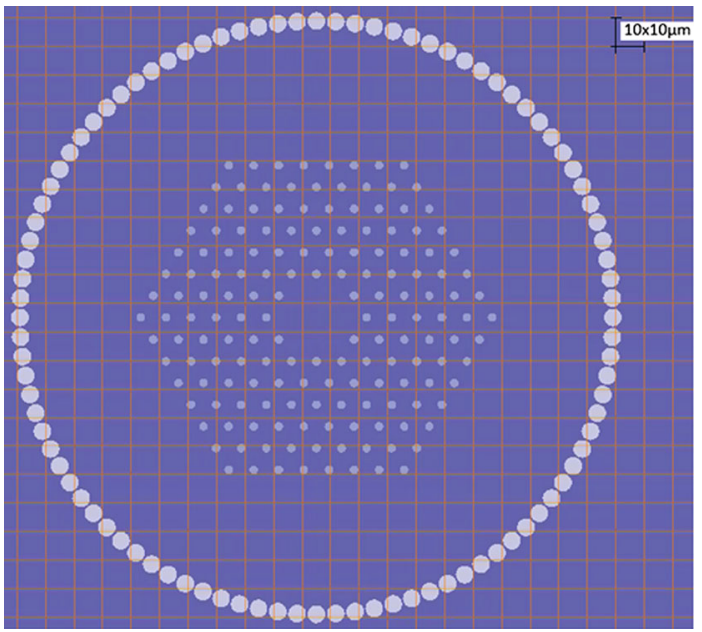



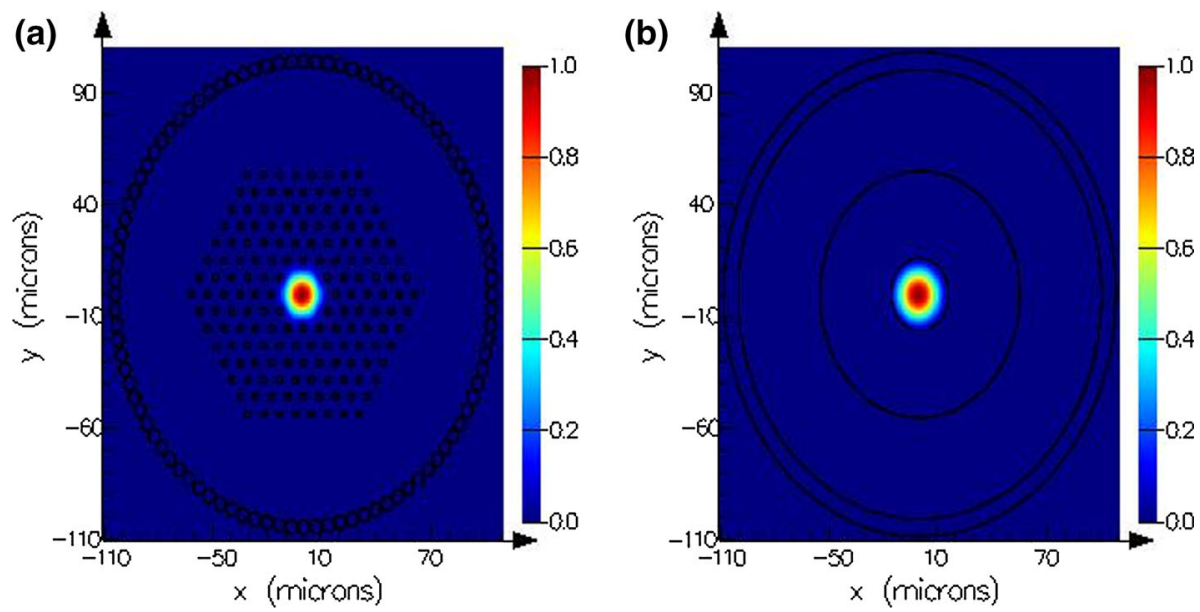

Fig. 4 Power distributions for fundamental modes calculated using the Lumerical Mode Solutions software for a real structure $\mathbf{b}$ approximated structure

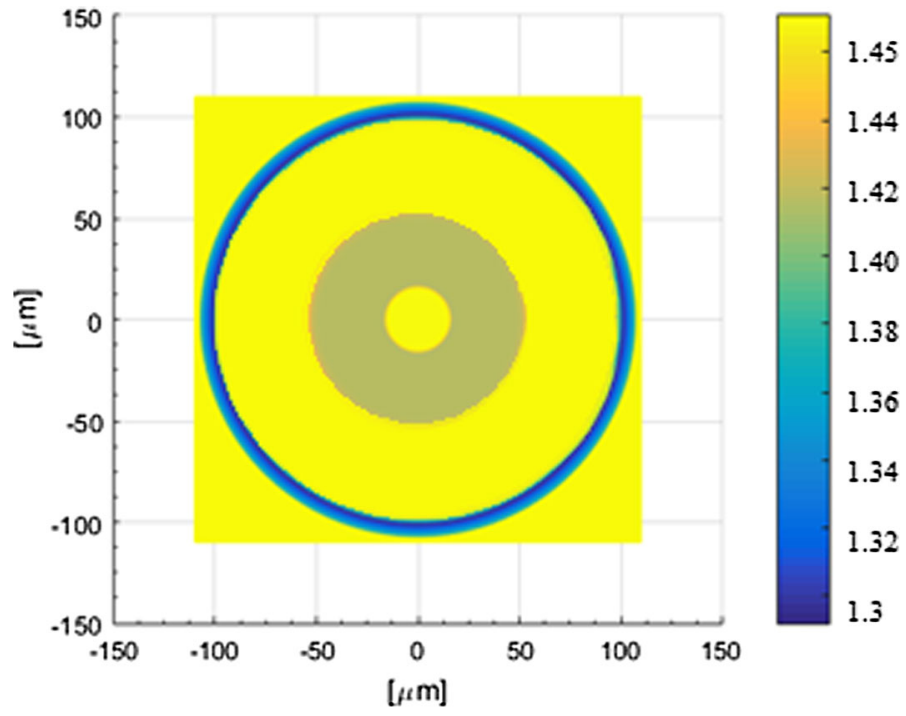

Fig. 5 Distribution of the refractive index determined for the investigated microstructured optical fibre with the use of Eq. 19

The working software for ray tracing simulation inside optical fibres was used to simulate ray tracing inside an optical coupler. A step-index multimode fibre 105/125 um was used as the pump fibre and the active fibre was a MOF with the earlier approximated layer structure (Fig. 5). The refractive index distribution for the multimode fibre was also approximated according to Eq. 19.

The first part of the analysis was the determination of ray tracing for MOFs. A beam of 10,000 rays, each at the angle from 0 to $5^{\circ}$ was introduced to a straight optical fibre (bending radius $\mathrm{R}=\infty$ ) in the area $0-100 \mu \mathrm{m}$ away from the core (both the core and 


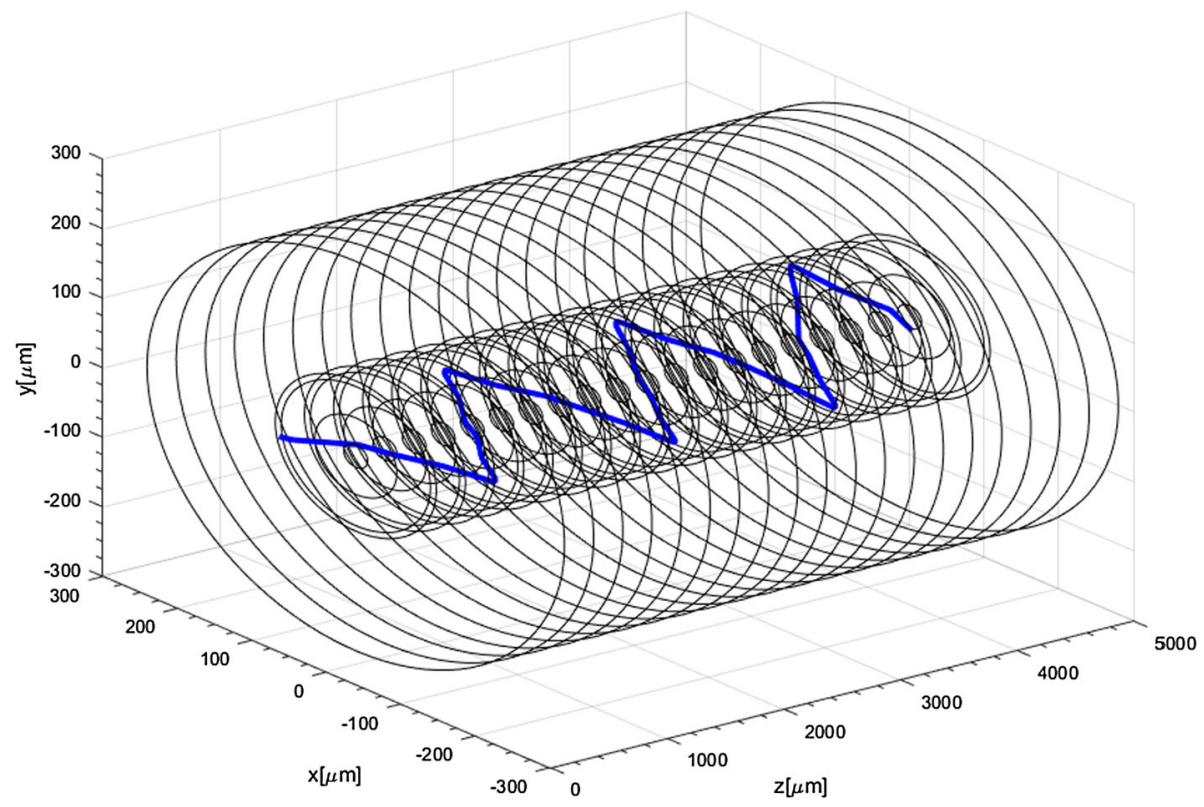

Fig. 6 Sample ray tracing through the modelled, straight MOF

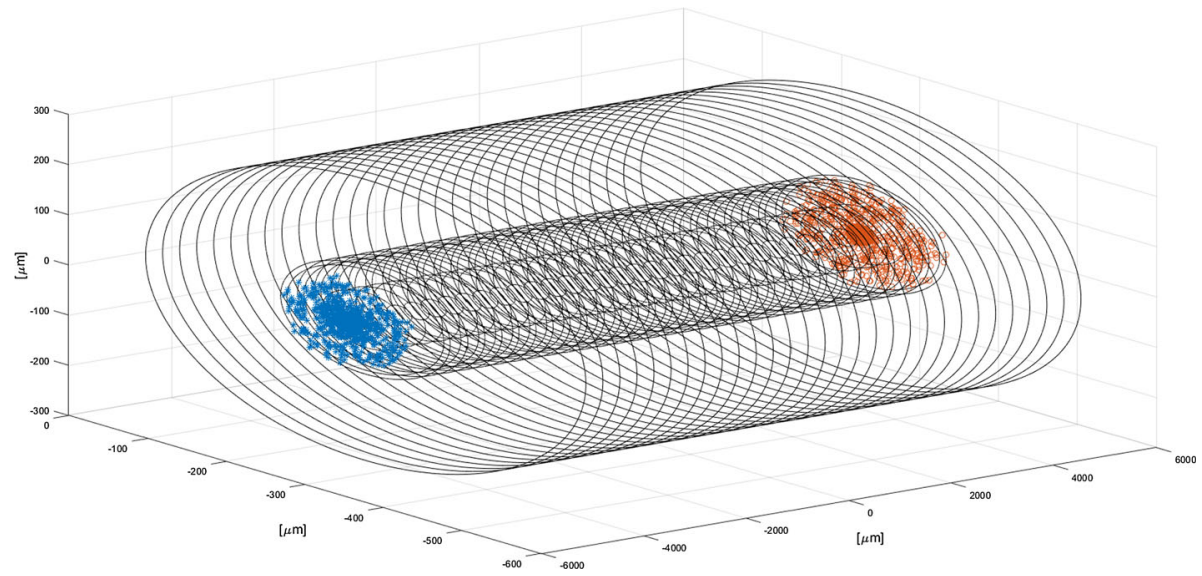

Fig. 7 Ray distribution inside the MOF on the input and after $1 \mathrm{~cm}$ section-3D projection

cladding were excited). It was assumed that the laser beam was strongly collimated and did not exceed the fibre NA to reduce the complexity of calculations. The normal distribution of the initial position of rays and angles was assumed. Sample ray tracing, ray distribution on the input and after crossing a $1 \mathrm{~cm}$ section of the MOF is presented in Figs. 6, 7 and 8.

The next step was the modelling of the optical fibre coupler made of a pump fibre $105 / 125 \mu \mathrm{m}$ and a MOF. The parameters of the designed optical fibre coupler depend not only on the structure of the fibres it is made of, but also on: polishing depth, bending angle of particular fibres, fibre shifting against one another and coupling parameters. The analysis of the influence of these parameters on ray tracing inside the coupler conducted in 

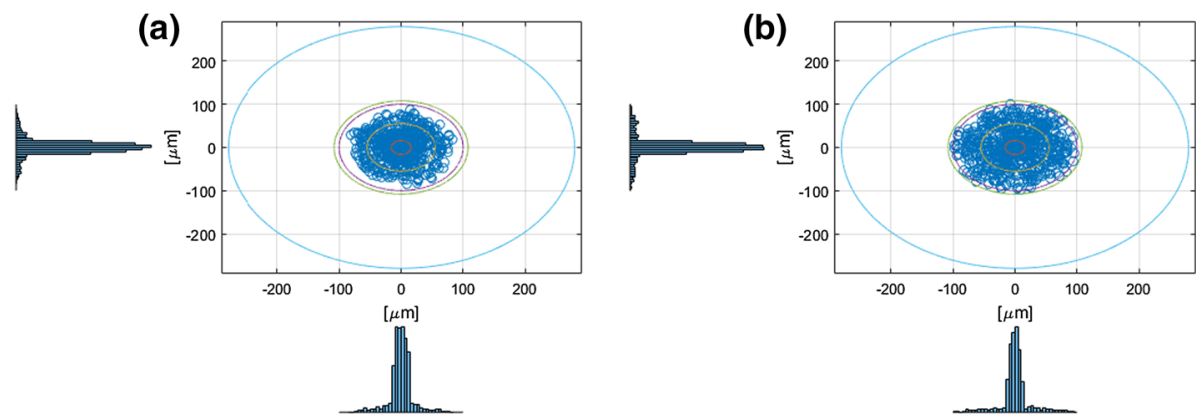

Fig. 8 Ray distribution inside MOF: a on the input, b after $1 \mathrm{~cm}$ section. The sections of ray distribution presented on the left and at the bottom of each distribution are treated as the pump power intensity distribution on the input (a) and after $1 \mathrm{~cm}$ fibre section (b)

simulation software can determine their optimum values. It is connected with enormous calculation power and time devoted to the simulation, however, it is by all means possible.

The article focuses only on showing that it is possible to use the ray-tracing method in the modelling of couplers made of MOF. The parameters of the simulated coupler were selected on the basis of the Authors' experience related to the construction couplers for conventional optical fibres. Hence, an optical fibre pumping $105 / 125 \mu \mathrm{m}$ was polished to the half way point in its core, while the MOF with a layered structure, presented in the earlier part of the article, was polished to the depth of $95 \mu \mathrm{m}$ from the core. The polishing value of the MOF was selected in such a way that the second cladding was affected only along the section of the connection with the pump fibre, which was to prevent additional leaks inside the coupler resulting from the mismatch between optical fibre sizes. To eliminate the air gap between the pumping fibre and the MOF, $3 \mu \mathrm{m}$ of a matching gel,

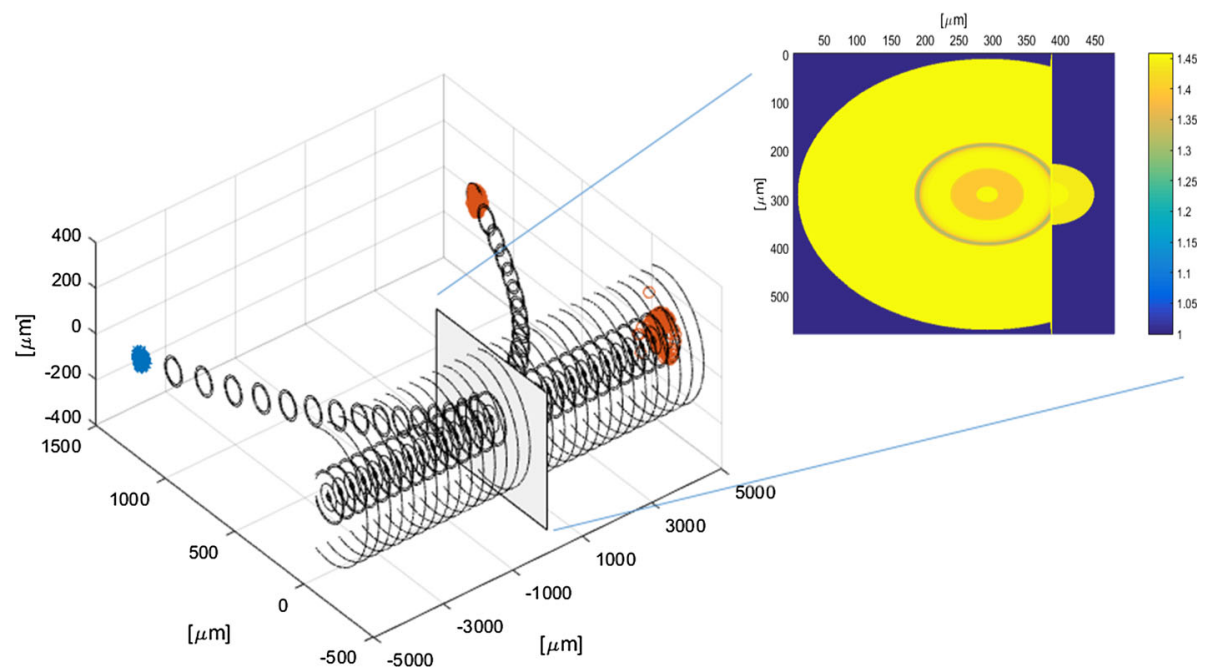

Fig. 9 Section of refractive index distribution across the centre of the coupler and the ray distribution on the input and output. The pump optical fibre $105 / 125 \mu \mathrm{m}$, bent on $1 \mathrm{~cm}$ radius, polished to the half point of its core; straight microstructure optical fibre, polished to $95 \mu \mathrm{m}$ from the core; $3 \mu \mathrm{m}$ of matching gel, $\mathrm{n}=1.4570$, between the optical fibres 

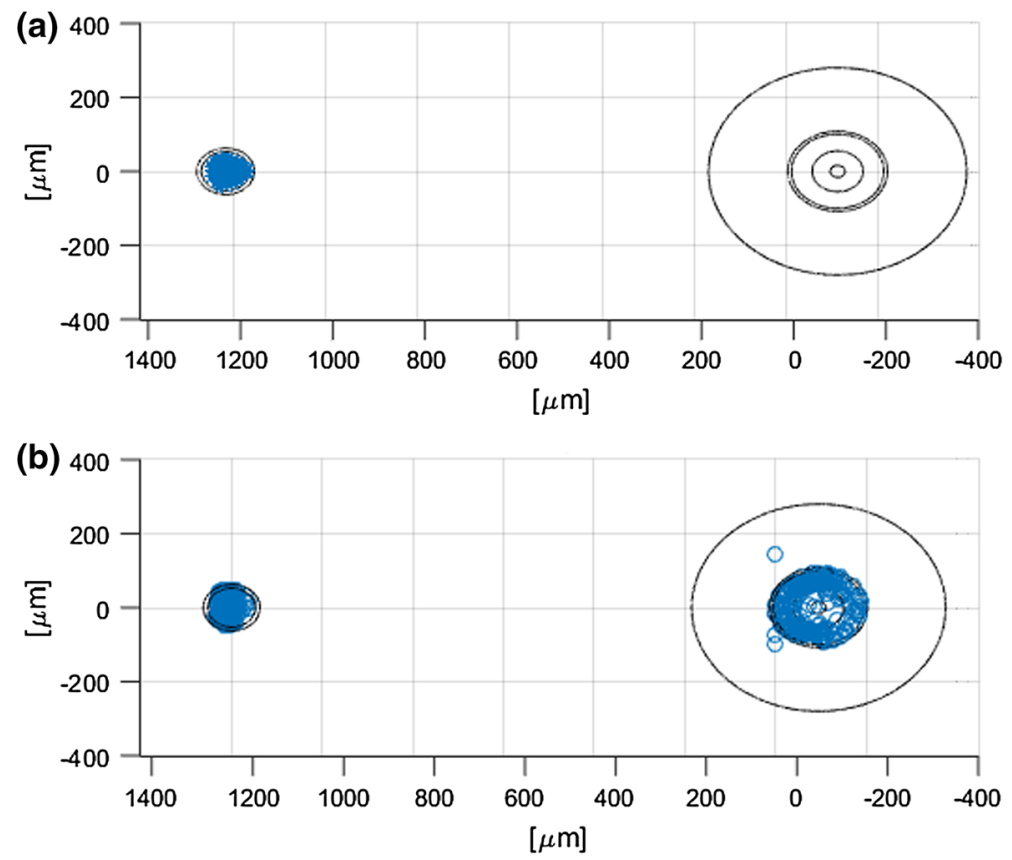

Fig. 10 Ray distribution on the input (a) and output (b) of a sample optical fibre coupler

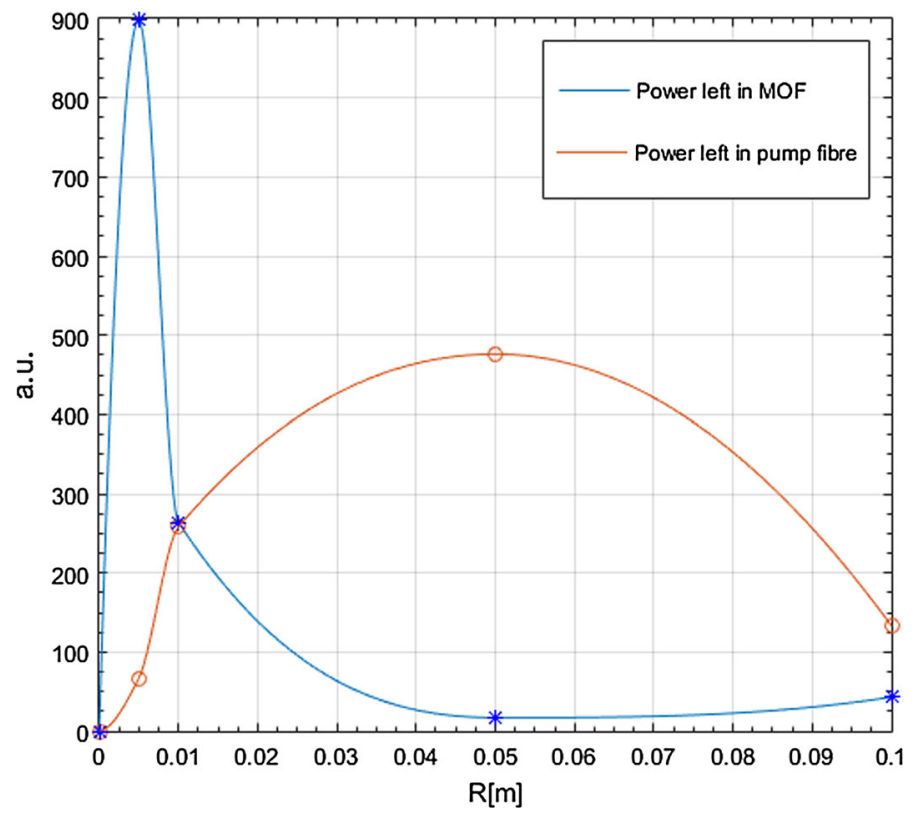

Fig. 11 Dependence between the number of rays moved to the MOF and remaining in the pump fibre and the bend radius of the pump fibre 
refractive index 1.4570, was placed between them. The thickness and the refractive index of its layer were determined on the basis of experiments conducted by the Authors on conventional optical fibres. Figures 9 and 10 presents the distribution of the rays introduced to and led out of a sample optical fibre coupler made of a MOF and a pump optical fibre $105 / 125 \mu \mathrm{m}$ and the section of refractive index distribution across the centre of the coupler.

For the purpose of demonstrating the benefits resulting from the use of this method, an optimisation attempt was made for one of the parameters of the above mentioned coupler. The MOF was straightened $(\mathrm{R}=\infty)$, while the pump fibre was bent at various radii. The other coupler parameters were not changed. As a result of the simulation the dependence between the number of rays moved to the MOF and staying in pump fibre and the bending radius $\mathrm{R}$ was obtained. The results are presented in Fig. 11.

With an appropriately low bend radius of the pump fibre, the majority of rays is introduced to the MOF. With an increase in the bend radius, more and more rays remain in the pump fibre. Thus, it was shown that the use of software allows to define the optimum parameters for an optical fibre coupler which could be used in pumping laser systems.

\section{Conclusions}

The article shows the application of the ray-tracing method for the approximation of raytracing through a DC MOF and optical fibre couplers based on microstructured fibres. The calculation was done for couplers made of well-defined fibres structures. To reduce the complexity of the structure the theory of an effective refractive index was used. By multiplying the number of rays and examining the distribution of the coupler output, it is possible to obtain the most important coupler parameters. After optimisation of their parameters, such couplers can be employed in laser systems that use a DC MOF as an active medium and a side pumping method to achieve the effective pumping. Moreover by utilization of the side-polishing technique in such lasers we can obtain the all-fibre laser cavity. The Authors are planning to continue the research to build real systems of optical fibre couplers. This will allow to verify the presented method and build fully-fiberized laser systems based on active microstructured fibres side-pumped with the use of optical fibre couplers made using the side-polishing technique.

Acknowledgements This work is co-financed by the Polish government as part of the Designated Subsidy for Young Scientists. The calculations were carried out in the Wroclaw Centre for Networking and Supercomputing (http://www.wcss.wroc.pl), Grant No. 184.

Open Access This article is distributed under the terms of the Creative Commons Attribution 4.0 International License (http://creativecommons.org/licenses/by/4.0/), which permits unrestricted use, distribution, and reproduction in any medium, provided you give appropriate credit to the original author(s) and the source, provide a link to the Creative Commons license, and indicate if changes were made.

\section{References}

Beres-Pawlik, E.: High-power laser. Polish patent, no. 217027 (2014)

Beres-Pawlik, E., Grobelny, A.: Construction of a side pumped double-clad fiber using polished couplers. Opt. Commun. 283, 2363-2368 (2010) 
Borbely, A., Johnson, S. G.: Prediction of light extraction efficiency of LEDs by ray trace simulation. In: Proceedings of SPIE 5187, Third International Conference on Solid State Lighting, vol. 301 (2004)

Heimrath, A.: A direct method for ray tracing in gradient-index fibres. J. Mod. Opt. 35(5), 783-790 (1988)

Horiuchi, S., Yoshida, S., Ushiyama, Z., Yamamoto, M.: Performance evaluation of GRIN lenses by ray tracing method. Opt. Quant. Electron. 42(2), 81-88 (2010)

Kim, H., Kim, J., Paek, U., Lee, B.H.: Tunable photonic crystal fiber coupler based on aside-polishing technique. Opt. Lett. 29(11), 1194-1196 (2004)

Lalau Keraly, C., Kuritzky, L., Cochet, M., Weisbuch, C.: Light extraction efficiency: a Ray tracing for light extraction efficiency (LEE) modeling in nitride LEDs. In: Seong, T.Y., Han, J., Amano, H., Morkoc, H. (eds.) III-Nitride Based Light Emitting Diodes and Applications Topics in Applied Physics, vol. 126, pp. 231-269. Springer, Netherlands Dordrecht (2013)

Sharma, A., Kumar, D.V., Ghatak, A.K.: Tracing rays through graded-index media: a new method. Appl. Opt. 21(6), 984-987 (1982)

Zervas, M.N., Codemard, C.A.: High power fiber lasers: a review. IEEE J. Sel. Topics Quantum Electron. 20(5), 219-241 (2014)

Zang, Z., Zeng, X., Du, J., Wang, M., Tang, X.: Femtosecond laser direct writing of microholes on roughened $\mathrm{ZnO}$ for output power enhancement of $\mathrm{InGaN}$ light-emitting diodes. Opt. Lett. 41, 3463-3466 (2016) 